\title{
The Role of Polyamine Metabolism in Neuronal Injury Following Cerebral Ischemia
}

\author{
Grace H. Kim*, Ricardo J. Komotar*, Margy E. McCullough-Hicks*, \\ Marc L. Otten, Robert M. Starke, Christopher P. Kellner, Matthew C. Garrett, \\ Maxwell B. Merkow, Michal Rynkowski, Kelly A. Dash, E. Sander Connolly \\ *Each of these authors contributed equally
}

\begin{abstract}
Stroke is a leading cause of morbidity and mortality in the US, with secondary damage following the initial insult contributing significantly to overall poor outcome. Prior investigations have shown that the metabolism of certain polyamines such as spermine, spermidine, and putrescine are elevated in ischemic parenchyma, resulting in an increase in their metabolite concentration. Polyamine metabolites tend to be cytotoxic, leading to neuronal injury in the penumbra following stroke and expansion of the area of infarcted tissue. Although the precise mechanism is unclear, the presence of reactive aldehydes produced through polyamine metabolism, such as 3-aminopropanal and acrolein, have been shown to correlate with the incidence of cerebral vasospasm, disruption of oxidative metabolism and mitochondrial functioning, and disturbance of cellular calcium ion channels. Regulation of the polyamine metabolic pathway, therefore, may have the potential to limit injury following cerebral ischemia. To this end, we review this pathway in detail with an emphasis on clinical applicability.
\end{abstract}

RÉSUMÉ: Le rôle du métabolisme de la polyamine dans le dommage neurologique dû à l'ischémie cérébrale. L'accident vasculaire cérébral (AVC) est une cause importante de morbidité et de mortalité aux États-Unis. Les dommages secondaires suite à l'accident initial contribuent significativement à l'issue défavorable. Des recherches antérieures ont démontré que le métabolisme de certaines polyamines comme la spermine, la spermidine et la putrescine est élevé dans le parenchyme ischémique, ce qui provoque une augmentation de la concentration de leurs métabolites. Les métabolites des polyamines sont généralement cytotoxiques, ce qui provoque des lésions neuronales pendant la pénombre ischémique suivant l'AVC et l'expansion de la zone infarcisée. Bien que le mécanisme précis ne soit pas clair, il a été démontré qu'il existe une corrélation entre la présence d'aldéhydes réactifs résultant du métabolisme de polyamines comme le 3-aminopropanal et l'acroléine, et l'incidence de vasospasme cérébral, la perturbation du métabolisme oxydatif et de la fonction mitochondriale, et la dysfonction des canaux calciques cellulaires. La régulation de la voie métabolique de la polyamine pourrait donc limiter l'ampleur de la lésion suite à une ischémie cérébrale. C'est dans ce but que nous revoyons cette voie métabolique en détail dans la perspective d'une applicabilité clinique éventuelle.

Can. J. Neurol. Sci. 2009; 36: 14-19

Stroke is the leading cause of disability and the third leading cause of death in the US, affecting more than 700,000 individuals each year. ${ }^{1}$ Moreover, the economic toll of stroke is profound, costing more than $\$ 33$ billion annually in direct health care fees and an additional $\$ 21$ billion in losses secondary to present treatment method inefficacies. ${ }^{1}$ Unfortunately there are few effective stroke therapies currently available.

Cell death in stroke is potentiated through a cascade of cytotoxins. In focal cerebral ischemia, cytotoxins travel past the immediate ischemic core and cause bystander cell death in the penumbra. ${ }^{2}$ While the ischemic core is generally believed to be unsalvageable, penumbral damage may be ameliorated if an effective intervention is instituted in a timely manner. ${ }^{1}$ To this end, research and development of therapeutic agents have focused on preserving the ischemic penumbra and limiting secondary injury following the initial insult.
Polyamines, such as putrescine, spermidine, and spermine are organic polycations that are present throughout the body and have widespread effects on enzymes, channels, and receptors. ${ }^{3}$ In the developing brain, polyamines are crucial for the regulation of cell division and are present in high concentrations. Once cells have matured and proliferation has ceased, polyamine levels drop and maintenance of homeostasis becomes critical. ${ }^{4}$

\footnotetext{
From the Department of Neurological Surgery, Columbia University, New York, New York, USA.

Received April 28, 2008. Final Revisions Submitted July 2, 2008. Correspondence to: Ricardo J. Komotar, Department of Neurosurgery, Columbia University, 710 West 168th Street, Room 431, New York, New York, 10032, USA.
} 
In addition to being associated with neuronal maturation, polyamines play a role in calcium flux regulation and glutamatergic receptor modulation within the adult central nervous system..$^{5-7}$ Polyamine functions are carried out via the polyamine interconversion cycle, the basic steps of which involve transformation of spermine into spermidine and spermidine into putrescine. ${ }^{8} \mathrm{~A}$ vital enzyme involved in these conversions is polyamine oxidase, whose structure and function is conserved across tissue types. ${ }^{8}$ Polyamines and their metabolic derivatives represent the majority of cytotoxins released following cerebral ischemia, contributing significantly to cell death. ${ }^{8}$ It follows, then, that the polyamine metabolic pathway represents a potential target for stroke therapy.

\section{Polyamine Metabolic Pathway}

The term "polyamine oxidase" or PAO has often been used to refer to a number of distinct enzymes involved in polyamine metabolism. It has been used to denote oxidase involvement in any reaction where acetyl polyamines or polyamines are oxidized and hydrogen peroxide is produced. ${ }^{9}$ Given the confusion over what actually is regarded as "polyamine oxidase," the following explanation of the polyamine interconversion pathway aims to clarify this terminology. The
Figure illustrates the polyamine metabolism pathway, including conversions of spermine (SPM) to spermidine (SPD) and spermidine to putrescine (PUT). These reactions occur both spontaneously and via the action of two major oxidases, polyamine oxidase (PAO) and serum amine oxidase (SAO). ${ }^{10}$

The first polyamine involved in the pathway, spermine, may go through any of three distinct, enzyme-catalyzed conversions, all of which ultimately result in the production of spermidine. The first path involves conversion of spermine to $\mathrm{N}$-acetyl spermine via an enzyme-catalyzed reaction of Spermine/ Spermidine N-acetyl Transferase (SSAT). ${ }^{11}$ This intermediate product then undergoes conversion to spermidine, hydrogen peroxide, and 3-acetoamidopropanal (3-AAP), catalyzed by PAOa. The second path involves direct conversion of spermine to spermidine and 3-aminopropanal (3-AP), catalyzed by PAOb. The third path involves conversion of spermine to spermine aldehyde via SAO catalysis. This intermediate product then undergoes spontaneous conversion to spermidine and acrolein.

Similarly, spermidine may undergo any of three different pathways to ultimately result in the production of putrescine. [Figure] The reactions that lead from spermidine to putrescine nearly mirror those of spermine to spermidine. The only notable differences are that the intermediates of the first and third

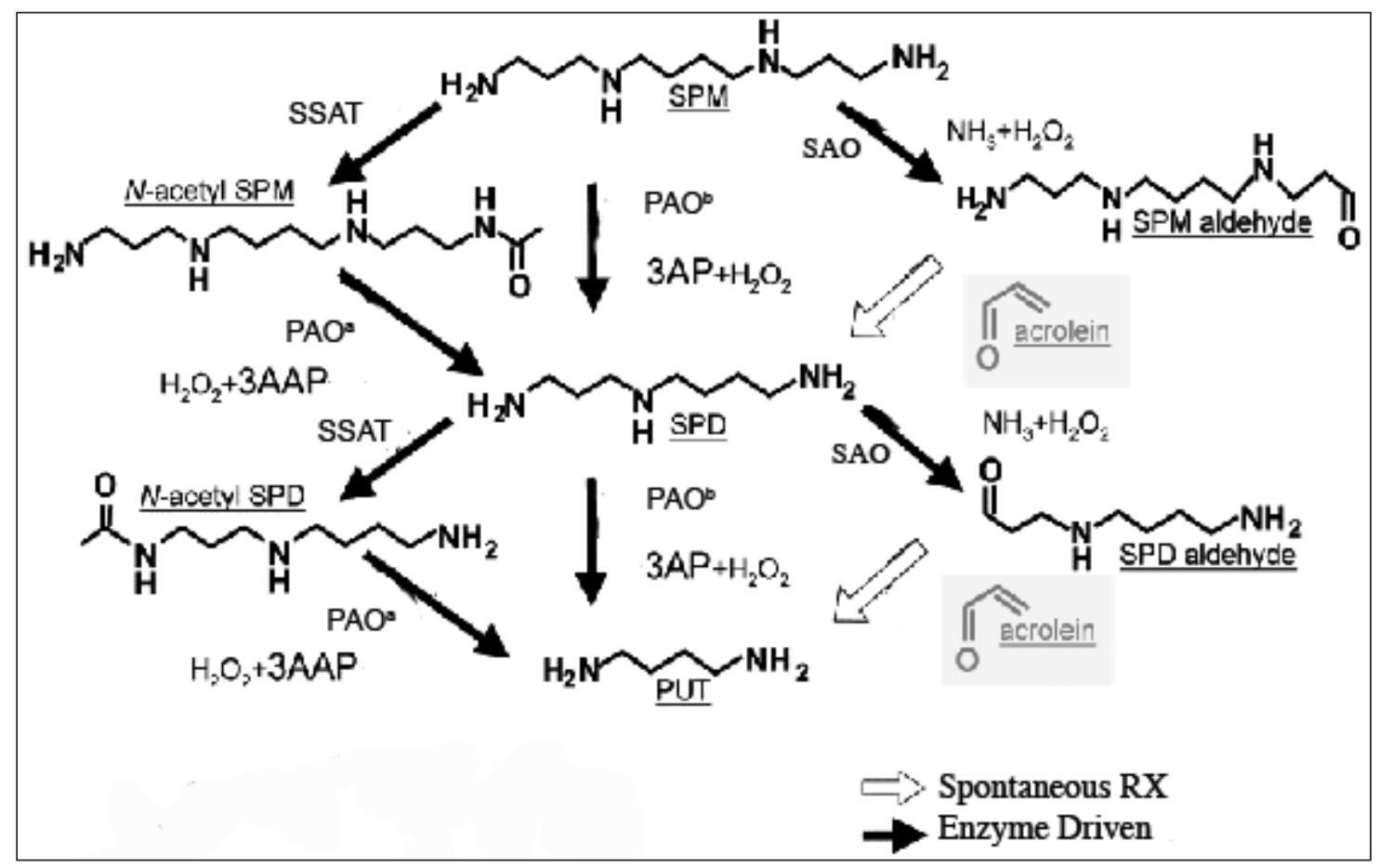

Figure: Polyamine Metabolic Pathway. SPM: Spermine; SPD: Spermidine; PUT: Putrescine; SSAT: Spermine/Spermidine $N$-acetyl Transferase; catalyzes transformation from Spermine to $N$-acetyl Spermine and Spermidine to $N$-acetyl Spermidine PAOa: Polyamine Oxidase a; catalyzes transformation from $\mathrm{N}$-acetyl Spermine to Spermidine and N-acetyl Spermidine to Putrescine; PAOb: Polyamine Oxidase b; catalyzes transformation from Spermine to Spermidine and Spermidine to Putrescine; SAO: Serum Amine Oxidase; catalyzes transformation from Spermine to Spermine aldehyde and Spermidine to Spermidine aldehyde 
aforementioned reactions are $\mathrm{N}$-acetyl spermidine and spermidine aldehyde, respectively.

The structural requirements for a substrate of PAO are two positively charged amino groups separated by a short carbon chain and an alkyl substituent on one or both nitrogen atoms. Spermine and spermidine are natural substrates for this enzyme. The catabolic pathway of polyamine metabolism that leads from spermine to spermidine and spermidine to putrescine relies on selective monoacetylation of the primary amino residues on spermine and spermidine, followed by oxidation of the $\mathrm{N}$ acetylaminopropyl terminus by PAO. ${ }^{8}$

Following the PAOb-catalyzed pathways from spermine to spermidine and spermidine to putrescine, the 3-AP byproducts spontaneously convert into acrolein and ammonia. Similarly, the 3-AAP that results as a byproduct from the PAOa catalyzed pathway may also spontaneously convert into acrolein and acetamide. It has been reported, though, that production of acrolein occurs less from 3-AAP than from 3-AP. ${ }^{12}$ Acrolein is spontaneously released from these aldehydes due to their significant instability at 37 degrees Celsius. ${ }^{13}$

Ivanova et al (1998) proposed that PAO directly oxidizes spermine and spermidine via oxidative deamination to produce 3-AP, hydrogen peroxide, and either spermidine or putrescine, respectively. This polyamine oxidase is denoted $\mathrm{PAOb}$, to distinguish it from PAOa which utilizes a different substrate. ${ }^{22}$

Tomitori et al use different terms for the same pathway designated in Figure 1. Instead of PAOa they refer to the enzyme that converts $\mathrm{N}$-acetyl spermine or spermidine "acetylpolyamine oxidase" or AcPAO..$^{12} \mathrm{PAOb}$ is referred to as "spermine oxidase" or SMO only in the reaction of spermine being oxidized to 3-AP, hydrogen peroxide, and spermidine. ${ }^{12}$ In yet another report, spermine oxidase is referred to as both SMO and hPAO- 1 and is responsible for spermine recycling within cells. ${ }^{14}$ Tomitori et al suggest that when spermine and acetylspermine are used as substrates, the terms "SAO" and "AcPAO" should be used to refer to the enzymes that partake in the polyamines' degradation. They do allow, however, for the possibility that other unnamed and unidentified amine oxidases may be involved in these two enzymatic activities. ${ }^{12}$

Seiler and colleagues categorize the various polyamine oxidases involved in eukaryotic polyamine metabolism into two different types. The first is copper-containing amine oxidases $(\mathrm{CuAO})$, which oxidatively deaminate the primary amino groups of polyamines to their corresponding aldehydes, an example of which is serum amine oxidase (SAO), ${ }^{8}, 15$ The second is flavinadenine dinucleotide (FAD)-dependent oxidase, like AcPAO, which is involved in the oxidative splitting of the monoacetyl derivatives of spermidine and spermine. ${ }^{8}$ In either case, these two delineations of polyamine metabolism ultimately refer to the same category of enzymes and compounds.

The reverse reaction, polyamine synthesis, is mediated primarily by ornithine decarboxylase (ODC) and S-adenosylmethionine decarboxylase (SAMDC). ${ }^{16}$ Ornithine decarboxylase is the enzyme responsible for catalyzing decarboxylation of the amino acid ornithine to putrescine, while SAMDC is the catalyst for the decarboxylation of S-adenosylmethionine. The decarboxylated form of S-adenosylmethionine provides an aminopropyl-moiety that is necessary for the interconversion of putrescine into spermidine and spermine. ${ }^{16}$
There is another pathway through which acrolein is produced that is not directly related to the aforementioned polyamine metabolic pathway but, nonetheless, results in the production of the aforementioned reactive aldehyde products. Adibhatla et al say that the activation of phospholipase A2 (PLA2) results in the hydrolysis of membrane phospholipids and the release of free fatty acids, including arachidonic acid. ${ }^{1}$ Oxidative metabolism of arachidonic acid generates reactive oxygen species (ROS), as does cardiolipin hydrolysis by mitochondrial secretory PLA2. Both of these processes contribute to the formation of lipid peroxides, which degrade to reactive aldehyde products, such as acrolein. ${ }^{1}$

\section{Polyamine Metabolism in Cerebral Ischemia}

Prior studies have demonstrated the involvement of polyamine metabolism in stroke, associating it with decreased levels of brain-tissue spermine and spermidine that follow from increased polyamine oxidation of these compounds, in addition to upregulated ODC activity. ${ }^{17}$ However, despite the fact that spermine and spermidine levels are declining in the ischemic brain, they may also simultaneously take serious effect, specifically demonstrated by evidence that spermine, spermidine, and polyamine oxidase are produced by damaged cells following cerebral ischemia and contribute to blood-brain barrier disturbances. ${ }^{12}$ Under normal conditions, the exchange between the brain and systemic circulation is limited, implying that deleterious changes in various protein levels in the context of cerebral ischemia may be secondary to this neuronal death and this subsequent blood-brain barrier incompetence. ${ }^{8}$

Ornithine decarboxylase is involved in only polyamine synthesis, but its activity during stroke has serious implications for subsequent polyamine breakdown. The ODC mRNA levels have been shown to increase after ischemia onset, indicating that the post-ischemic ODC rise in activity results from an activation of gene expression, most pronounced in the hippocampus. ${ }^{18}$ It has been shown that activation of ODC during cerebral ischemia produces a considerable increase in putrescine content, while spermidine and spermine levels remain constant or decline. In normal brain, increased ODC activity results in elevation of all three polyamines. ${ }^{18}$ The difference between ischemic and normal brain polyamine levels is due to a marked reduction in SAMDC activity following ischemia; SAMDC is rate-limiting in the synthesis of spermidine and spermine, so the conversion of putrescine to these two other polyamines is significantly downregulated during ischemic periods. ${ }^{19}$ Furthermore, putrescine elevation in ischemic brain may be attributed to impaired clearance..$^{20}$ Paschen et al examined putrescine in the ischemic brain, and found that 30 minutes after cerebral ischemia, ODC activity is induced 10-fold leading to increased levels of putrescine..$^{20}$ Based on the following findings, examining putrescine levels in conjunction with ODC activity may be a more accurate indicator of pathology than isolated ODC activity: ${ }^{16} 1$. Stroke duration correlates with the increase of putrescine following onset of ischemia. 2. An increase in putrescine levels precedes neuronal necrosis. 3. Neuronal necrosis correlates with putrescine levels.

In addition to putrescine, cytotoxic aldehydes, such as 3aminopropanal and acrolein, increase as well following cerebral ischemia. During cerebral ischemia, cells in the densely hypoxic 
core release stores of intracellular spermine and spermidine, which are later catabolized by PAO to produce reactive aldehydes. These products then go on to cause apoptosis and necrosis in surrounding glia and neurons, thus promoting continued release of spermine and spermidine. ${ }^{21}$ The major catabolic products of PAO metabolism of spermine and spermidine are putrescine and 3-aminopropanal. It has been shown that neurotoxic levels of 3-AP accumulate within two hours of forebrain ischemia induction in rats. ${ }^{22} 3$-AP levels are also elevated in the cerebrospinal fluid of humans with CNS ischemia. Lastly, 3-AP levels in the brain continue to increase for at least 25 hours after the onset of cerebral ischemia, corresponding to spreading neuronal and glial injury. ${ }^{2}$

Acrolein levels are also increased after onset of cerebral ischemia, secondary to elevated PAO activity. Wood et al reported that acrolein, in addition to 3-AP, was present at high levels in rat stroke models, with delayed penumbral cell death being preceded by increases in these reactive aminoaldehydes. ${ }^{19}$ In another study, examining the presence of acrolein in guinea pig spinal cords, acrolein was present on axons following ischemic insult. ${ }^{23}$ Clinical studies have demonstrated levels of acrolein, among other polyamine metabolic byproducts, to be significantly elevated in patients following cerebral ischemia. In addition, stroke severity paralleled the acrolein level and total polyamine oxidase (AcPAO plus SMO) in these patients. ${ }^{12}$ The same study measured, via high-performance liquid chromatography (HPLC), the levels of acetylpolyamine oxidase and spermine oxidase (SMO) and the levels of protein conjugated acrolein via Enzyme-linked immunosorbent assay (ELISA) in the plasma of stroke patients. ${ }^{12}$ Following onset of cerebral ischemia, AcPAO increased first, followed by higher levels of SMO, and finally an increase in acrolein. These results support the idea that AcPAO and SMO are released from cells shortly after stroke, leading to lower amounts of spermine and spermidine and increased levels of acrolein. ${ }^{12}$

As aforementioned, acrolein levels also increase following the activation of PLA2. ${ }^{1}$ Adibhatla et al cite that following transient cerebral ischemia, both lipid peroxidation and activation of PLA2 are increased, leading to an increase of acrolein and other less reactive aldehyde products, such as malondialdehyde (MDA) and 4-hydroxynonenal (NHE). ${ }^{1}$

\section{Mechanism of PAO Mediated Cytotoxicity in Ischemic Brain}

One of the primary ways polyamine oxidation causes neuronal damage is via reactive aldehyde intermediates that directly mediate cytotoxicity. ${ }^{17}$ As previously discussed, cerebral ischemia stimulates increased PAO activity, polyamine catabolism, and reactive aldehyde production. The final result is neuronal cell necrosis and glial cell apoptosis. ${ }^{2}$ There is a loss of cell compartmentalization during cerebral ischemia, allowing release of 3-AP that goes on to bind to structural membrane proteins and alter critical functions. ${ }^{2} 3$-AP production during cerebral ischemia increases before onset of significant cellular degeneration and continues to rise during cell death. ${ }^{22} 3$-AP is thought, as a reactive aldehyde, to interact with important cellular proteins' amino and thiol groups, compromising their important functions. ${ }^{24}$ In glial cells, for example, 3-AP mediates apoptosis by accumulating in lysosomes, causing lysosomic rupture, and inducing activation of a caspase-1 dependent signaling pathway. ${ }^{19,22}$ In neurons, 3-AP has been shown to cause necrotic death. ${ }^{22}$ The amino and aldehyde functions of 3-AP are required for cytotoxicity. The amino group confers lysosomotropism while the aldehyde group has unknown, yet important, functions. ${ }^{24}$ Goodenough et al studied the effect of direct injection of polyamines into the central nervous system of rats and found that direct injection of spermine produced a large lesion that showed, upon DNA analysis, signs of degradation often associated with apoptosis, thus confirming that polyamine metabolism leads to apoptotic cell death. ${ }^{3}$

A second hypothesis regarding how polyamines and their oxidative products may induce cell death during ischemia involves vasospasm. When blood is injected into the subarachnoid space, it consistently causes acute and chronic vasospasm of cerebral arteries secondary to endothelial irritation. ${ }^{25}$ Acrolein and other polyamine metabolites are likely candidates for mediators of cerebral vasospasm. ${ }^{26}$ When either acrolein or allylamine (AA), a three-carbon amine and precursor to acrolein production in other body tissues, comes in direct contact with rat coronary artery or thoracic artery, hypercontractility occurs. ${ }^{26}$ Following metabolism of AA to acrolein, hydrogen peroxide, and ammonia by coronary artery semicarbazinde-sensitive amine oxidase (SSAO), acrolein likely induces vasospasm independent of, or in addition to, an indirect mechanism through endothelial injury. ${ }^{26}$ Acrolein has been shown to induce contraction in a number of different types of smooth muscle, including isolated tracheal smooth muscle and isolated vas deferens smooth muscle. ${ }^{26}$ By stimulating vasospasm, then, acrolein contributes to significant secondary injury following ischemia.

The precise mechanism of acrolein-mediated cytotoxicity, however, remains unclear. Following exposure of PC12 cells to various concentrations of acrolein, higher concentrations (around $100 \mathrm{mM}$ ) were shown to produce consistent cell death and complete collapse of mitochondrial functioning. ${ }^{27} \mathrm{By}$ four hours, microtubules were disassembled, degraded, and dissolved, leaving little cytoplasm. ${ }^{27}$ In addition, acrolein was shown to accumulate in the cytoplasm, have a longer half-life than many other cytotoxic agents, and pass through undamaged cell membranes, leading to further neuronal damage. ${ }^{28}$ The conclusions drawn from these studies were the following: 1) Acrolein is a potent toxin capable of killing entire PC12 populations within 12 hours. 2) Collapse of oxidative metabolism by mitochondria is significant after acrolein exposure. 3) Calpain, the key enzyme responsible for microtubule degradation, is upregulated after acrolein exposure. 4) Cell death from acrolein is likely due to necrosis, as opposed to apoptosis, in these cells. This final conclusion was based upon the fact that caspase 3 was not upregulated and there was no detection of DNA laddering after exposure to acrolein. ${ }^{27}$ It has been shown, however, that acrolein in low concentrations can induce apoptotic death in various cell types, such as Chinese hamster ovary cells, ${ }^{29}$ neutrophils ${ }^{30}$ and human alveolar macrophages. ${ }^{31}$

Other studies have also investigated the specific mechanism by which acrolein conveys cytotoxicity. One study proposed that acrolein inhibits mitochondrial respiration directly. ${ }^{32}$ A second possible mechanism is the inhibition of respiration by the formation of a Michael adduct of acrolein with mitochondrial 
proteins, as studied by Zollner et al. ${ }^{33}$ Yet another theory is that acrolein indirectly inhibits respiration by uncoupling oxidative phosphorylation and ATP production. ${ }^{34,35}$ While no conclusions have yet been made on the specific mode by which acrolein confers cytotoxicity, it does appear to be attributable to adverse effects upon mitochondria.

Another possible way by which polyamine metabolites confer cytotoxicity is through calcium ion disturbance. As previously discussed, post-ischemic activation of ODC and inhibition of Sadenosylmethionine decarboxylase are responsible for the increase of putrescine, which has been implicated in delayed neuronal death after ischemia. ${ }^{20}$ This cytotoxicity may occur via $\mathrm{Ca}^{2+}$ disturbances secondary to putrescine buildup and subsequent release of excitatory amino acids. ${ }^{20}$ Electrical hyperactivity follows, leading to cell death, particularly within the penumbra. ${ }^{20}$ It is evident, therefore, that the metabolites of polyamines following cerebral ischemia are cytotoxic and responsible for much of the secondary injury in stroke patients. While these metabolites may have limited negative effects in isolation, they lead to devastating effects on brain tissue in combination.

\section{Inhibition of Polyamine Cytotoxicity in Cerebral Ischemia}

Numerous studies have manipulated polyamine metabolism in efforts to ameliorate its deleterious effects following cerebral ischemia. Wallace et al examined polyamine analogues, which can act on multiple targets, such as the down regulation of polyamine synthesis through inhibition of ODC, inhibition of SAMDC, and decreasing uptake of polyamines into the blood. ${ }^{14}$ Both difluoromethylornithine and methylglyoxal-bis-(guanylhydrazone) are effective compounds for targeting polyamine synthesis. Difluoromethylornithine is an inhibitor of ODC, FDAapproved, and leads to decreased spermidine content in addition to lower ODC acivity. ${ }^{14}$ Methylglyoxal-bis-(guanyl-hydrazone) is a competitive inhibitor of SAMC and depletes spermidine and spermine content in tissue where polyamine synthesis is upregulated. ${ }^{14}$

Cockroft et al found that aminoguanidine offered cerebroprotection in a rodent model of stroke in addition to halting cytotoxic effects of polyamines. ${ }^{17}$ They proposed that aminoguanidine's therapeutic effects may be due to inhibition of toxic aldehyde intermediates that arise when PAO and its substrates are elevated following onset of ischemia. ${ }^{17}$

A third study found that N-2-mercaptoglycine (N-2-MPG) reacts with 3-AP to form a nontoxic thioacetal adduct that cannot accumulate in and cause rupture of lysosomes. ${ }^{2}$ They report that different inhibitors of polyamine oxidases, such as N-2-MPG, are successful in their actions to prevent cytotoxicity by reducing the production of various reactive aldehydes and protect against development of brain damage in rats with focal cerebral ischemia. $^{2}$

Ivanova et al examined rats treated with two structurally different inhibitors of PAO, aminoguanidine and chloroquine. ${ }^{22}$ They found that both of these compounds attenuated brain PAO activity, preventing the production of 3-AP and protecting the brain against ischemic damage. ${ }^{22}$

Wood et al showed that hydroxylamines conferred cerebroprotection by inhibiting aldehyde cytotoxicity following cerebral ischemia. They hypothesized that if aldehydes were, in fact, important mediators of cerebral injury, then a drug that could buffer reactive aldehydes would be superior to previously tested agents. ${ }^{19}$ The mechanism by which hydroxylamines inactivate these aldehydes was thought to be through formation of oximes with aldehydes in the cytosol and subsequent entry into lysosomes as a result of their basic nitrogen group; these oximes were thought to inactivate the aldehydes within the cellular compartment. ${ }^{19}$

A recent study by Liu-Snyder et al tested the efficacy of hydralazine in PC12 cells exposed to acrolein. In the presence of acrolein, hydralazine has been shown to form hydrazone adducts, which reduce acrolein toxicity and protects PC12 cells from death. ${ }^{28}$ Hydralazine's nitrogen component allows the compound to react with acrolein, leading to "aldehyde trapping" that significantly decreases the cytotoxicity of the aldehyde within cells. ${ }^{28}$

Hurtado et al studied the effect of a chronic treatment with CDP-choline following cerebral ischemia. ${ }^{36} \mathrm{CDP}$-choline is an intermediate in membrane phospholipids synthesis that, when administered exogenously, increases phosphatidylcholine and sphingomyelin membrane content on the first day of reperfusion after stroke. Hurtado et al found that CDP-choline, when administered 24 hours after the onset of ischemia in adult male Sprague-Dawley rats, stimulates glutathione synthesis and decreases lipid peroxidation, which results in both a decreased rate of acrolein synthesis and has also been shown to increase synaptic plasticity. Hurtado et al concluded that animals treated with CDP-choline showed enhanced dendritic complexity and spine density as compared with the control group, suggesting that chronic treatment with CDP-choline initiated 24 hours after the insult is able to increase the neuronal plasticity within noninjured and functionally connected brain regions, as well as to promote functional recovery. ${ }^{36}$ Additionally, Adibhatla et al cite that CDP-choline has been shown to affect PLA2 activation and attenuates increased PLA2 activity and formation of malondialdehyde following transient forebrain ischemia. ${ }^{1}$

\section{Conclusions}

The study of polyamine metabolism in cerebral ischemia holds much promise, as there is a consensus on the fact that polyamine metabolites do confer cytotoxicity and thus secondary injury in the ischemic brain. While studies have tested various compounds' potential effectiveness in attenuating cerebral injury in cell cultures and animal models, few clinical trials have been initiated. Future studies identifying similar therapeutic compounds and designing appropriate clinical trials will be critical to advancing stroke management.

\section{REFERENCES}

1. Adibhatla RM, Hatcher JF, Dempsey RJ. Phospholipase A2, hydroxyl radicals, and lipid peroxidation in transient cerebral ischemia. Antioxid Redox Signal. 2003 Oct;5(5):647-54.

2. Ivanova S, Batliwalla F, Mocco J, Kiss S, Huang J, Mack W, et al. Neuroprotection in cerebral ischemia by neutralization of 3aminopropanal. Proc Natl Acad Sci USA. 2002 Apr 16;99(8): 5579-84.

3. Goodenough S, Davidson M, Kidd G, Matsumoto I, Wilce P. Cell death and immunohistochemistry of p53, c-Fos and c-Jun after spermine injection into the rat striatum. Exp Brain Res. 2000 Mar;131(1):126-34. 
4. Slotkin TA, Bartolome J. Role of ornithine decarboxylase and the polyamines in nervous system development: a review. Brain Res Bull. 1986 Sep;17(3):307-20.

5. Iqbal Z, Koenig H. Polyamines appear to be second messengers in mediating $\mathrm{Ca} 2+$ fluxes and neurotransmitter release in potassium-depolarized synaptosomes. Biochem Biophys Res Commun. 1985 Dec 17;133(2):563-73.

6. Rock DM, Macdonald RL. Polyamine regulation of N-methyl-Daspartate receptor channels. Annu Rev Pharmacol Toxicol. 1995; 35:463-82.

7. Williams K, Romano C, Molinoff PB. Effects of polyamines on the binding of $[3 \mathrm{H}] \mathrm{MK}-801$ to the $\mathrm{N}$-methyl-D-aspartate receptor: pharmacological evidence for the existence of a polyamine recognition site. Mol Pharmacol. 1989 Oct;36(4):575-81.

8. Seiler N. Polyamine oxidase, properties and functions. Prog Brain Res. 1995;106:333-44.

9. Ferioli ME, Berselli D, Caimi S. Effect of mitoguazone on polyamine oxidase activity in rat liver. Toxicol Appl Pharmacol. 2004 Dec 1;201(2):105-11

10. Takano K, Ogura M, Yoneda Y, Nakamura Y. Oxidative metabolites are involved in polyamine-induced microglial cell death. Neuroscience. 2005;134(4):1123-31

11. Nagesh Babu G, Sailor KA, Sun D, Dempsey RJ. Spermidine/ spermine $\mathrm{N} 1$-acetyl transferase activity in rat brain following transient focal cerebral ischemia and reperfusion. Neurosci Lett. 2001 Mar 2;300(1):17-20.

12. Tomitori H, Usui T, Saeki N, Ueda S, Kase H, Nishimura K, et al. Polyamine oxidase and acrolein as novel biochemical markers for diagnosis of cerebral stroke. Stroke. 2005 Dec;36(12): 2609-13.

13. Alarcon RA. Acrolein. IV. Evidence for the formation of the cytotoxic aldehyde acrolein from enzymatically oxidized spermine or spermidine. Arch Biochem Biophys. 1970 Apr; 137(2):365-72.

14. Wallace HM, Fraser AV. Inhibitors of polyamine metabolism: review article. Amino Acids. 2004 Jul;26(4):353-65.

15. Seiler N. Catabolism of polyamines. Amino Acids. 2004 Jun;26(3): 217-33.

16. Paschen W. Polyamine metabolism in different pathological states of the brain. Mol Chem Neuropathol. 1992 Jun;16(3):241-71.

17. Cockroft KM, Meistrell M, 3rd,Zimmerman GA, Risucci D, Bloom $\mathrm{O}$, Cerami A, et al. Cerebroprotective effects of aminoguanidine in a rodent model of stroke. Stroke. 1996 Aug;27(8):1393-8.

18. Temiz C, Dogan A, Baskaya MK, Dempsey RJ. Effect of difluoromethylornithine on reperfusion injury after temporary middle cerebral artery occlusion. J Clin Neurosci. 2005 May;12(4): 449-52.

19. Wood PL, Khan MA, Kulow SR, Mahmood SA, Moskal JR. Neurotoxicity of reactive aldehydes: the concept of "aldehyde load" as demonstrated by neuroprotection with hydroxylamines. Brain Res. 2006 Jun 20;1095(1):190-9.

20. Paschen W, Schmidt-Kastner R, Djuricic B, Meese C, Linn F, Hossmann KA. Polyamine changes in reversible cerebral ischemia. J Neurochem. 1987 Jul;49(1):35-7.
21. Takano K, Ogura M, Nakamura Y, Yoneda Y. Neuronal and glial responses to polyamines in the ischemic brain. Curr Neurovasc Res. 2005 Jul;2(3):213-23.

22. Ivanova S, Botchkina GI, Al-Abed Y, Meistrell M, 3rd, Batliwalla F, Dubinsky JM, et al. Cerebral ischemia enhances polyamine oxidation: identification of enzymatically formed 3-aminopropanal as an endogenous mediator of neuronal and glial cell death. J Exp Med. 1998 Jul 20;188(2):327-40.

23. Peasley MA, Shi R. Ischemic insult exacerbates acrolein-induced conduction loss and axonal membrane disruption in guinea pig spinal cord white matter. J Neurol Sci. 2003 Dec 15;216(1): 23-32.

24. Li W, Yuan XM, Ivanova S, Tracey KJ, Eaton JW, Brunk UT. 3Aminopropanal, formed during cerebral ischaemia, is a potent lysosomotropic neurotoxin. Biochem J. 2003 Apr 15;371(Pt 2): 429-36.

25. Echlin F. Experimental vasospasm, acute and chronic, due to blood in the subarachnoid space. J Neurosurg. 1971 Dec;35(6):646-56.

26. Conklin DJ, Boyce CL, Trent MB, Boor PJ. Amine metabolism: a novel path to coronary artery vasospasm. Toxicol Appl Pharmacol. 2001 Sep 1;175(2):149-59.

27. Liu-Snyder P, McNally H, Shi R, Borgens RB. Acrolein-mediated mechanisms of neuronal death. J Neurosci Res. 2006 Jul;84(1): 209-18.

28. Liu-Snyder P, Borgens RB, Shi R. Hydralazine rescues PC12 cells from acrolein-mediated death. J Neurosci Res. 2006 Jul;84(1): 219-27.

29. Tanel A, Averill-Bates DA. P38 and ERK mitogen-activated protein kinases mediate acrolein-induced apoptosis in Chinese hamster ovary cells. Cell Signal. 2007 May;19(5):968-77.

30. Finkelstein EI, Nardini M, van der Vliet A. Inhibition of neutrophil apoptosis by acrolein: a mechanism of tobacco-related lung disease? Am J Physiol Lung Cell Mol Physiol. 2001 Sep;281(3): L732-9.

31. Li L, Hamilton RF, Jr., Taylor DE, Holian A. Acrolein-induced cell death in human alveolar macrophages. Toxicol Appl Pharmacol. 1997 Aug;145(2):331-9.

32. Picklo MJ, Montine TJ. Acrolein inhibits respiration in isolated brain mitochondria. Biochim Biophys Acta. 2001 Feb 14;1535 (2):145-52.

33. Zollner H. Inhibition of some mitochondrial functions by acrolein and methylvinylketone. Biochem Pharmacol. 1973 May 15;22 (10): $1171-8$

34. Harper ME, Bevilacqua L, Hagopian K, Weindruch R, Ramsey JJ. Ageing, oxidative stress, and mitochondrial uncoupling. Acta Physiol Scand. 2004 Dec;182(4):321-31.

35. Luo J, Shi R. Acrolein induces oxidative stress in brain mitochondria. Neurochem Int. 2005 Feb;46(3):243-52.

36. Hurtado O, Cardenas A, Pradillo JM, Morales JR, Ortego F, Sobrino $\mathrm{T}$, et al. A chronic treatment with CDP-choline improves functional recovery and increases neuronal plasticity after experimental stroke. Neurobiol Dis. 2007 Apr;26(1): 105-11. 